\title{
The association of serum testosterone levels and ventricular repolarization
}

\author{
Charlotte van Noord • Marcus Dörr • Miriam C. J. M. Sturkenboom • \\ Sabine M. J. M. Straus • Thorsten Reffelmann - Stephan B. Felix • \\ Albert Hofman • Jan A. Kors • Robin Haring • Frank H. de Jong • \\ Matthias Nauck • André G. Uitterlinden - Henri Wallaschofski • \\ Jacqueline C. M. Witteman • Henry Völzke • Bruno H. Ch. Stricker
}

Received: 7 July 2009/Accepted: 19 November 2009/Published online: 3 December 2009

(C) The Author(s) 2009. This article is published with open access at Springerlink.com

\begin{abstract}
It is assumed that testosterone is an important regulator of gender-related differences in ventricular repolarization. Therefore, our aim was to study whether serum levels of testosterone are associated with QTc, QT and RR interval variation. Setting: two independent population-based cohort studies. Participants: 445 male participants ( $\geq 55$ years) from the Rotterdam study cohort and 1,428 male participants from the study of health in Pomerania (SHIP) with an electrocardiogram who were randomly sampled for assessment of serum testosterone at baseline, after exclusion of participants with testosterone
\end{abstract}

C. van Noord - M. C. J. M. Sturkenboom .

S. M. J. M. Straus - A. Hofman - A. G. Uitterlinden -

J. C. M. Witteman - B. H. Ch. Stricker ( $\square)$

Department of Epidemiology, Erasmus Medical Center,

Rotterdam, The Netherlands

e-mail: b.stricker@erasmusmc.nl

C. van Noord

e-mail: c.vannoord@erasmusmc.nl

M. Dörr · T. Reffelmann - S. B. Felix · R. Haring · M. Nauck · H. Wallaschofski $\cdot$ H. Völzke

Ernst Moritz Arndt University Greifswald, Greifswald, Germany

F. H. de Jong - A. G. Uitterlinden - B. H. Ch. Stricker Department of Internal Medicine, Erasmus Medical Center, Rotterdam, The Netherlands

\section{C. J. M. Sturkenboom - J. A. Kors}

Department of Medical Informatics, Erasmus Medical Center, Rotterdam, The Netherlands

C. van Noord · S. M. J. M. Straus

The Dutch Medicines Evaluation Board, The Hague,

The Netherlands

B. H. Ch. Stricker

The Inspectorate for Health Care, The Hague, The Netherlands altering drugs, QTc prolonging drugs or dig(it)oxin, left ventricular hypertrophy and left and right bundle branch block. Endpoints: length of the QTc, QT and RR intervals. Analysis: linear regression model, adjusted for the two individual studies and a pooled analysis of both studies. The pooled analysis of the Rotterdam study and SHIP showed that the QTc interval gradually decreased among the tertiles ( $P$ value for trend 0.024). The third tertile of serum testosterone was associated with a lower QTc interval compared to the first tertile $[-3.4 \mathrm{~ms}(-6.5$; $-0.3)]$. However, the third tertile of serum testosterone was not associated with a lower QT interval compared to the first tertile $[-0.7 \mathrm{~ms}(-3.1 ; 1.8)]$. The RR interval gradually increased among the tertiles ( $P$ value for trend 0.002 ) and the third tertile of serum testosterone showed an increased RR interval compared to the first tertile $[33.5 \mathrm{~ms}$ $(12.2 ; 54.8)]$. In the pooled analysis of two populationbased studies, serum testosterone levels were not associated with the QT interval, which could be due to a lack of power. Lower QTc intervals in men with higher serum testosterone levels could be due to the association of serum testosterone with prolongation of the RR interval.

Keywords Serum testosterone - Ventricular repolarization $\cdot$ QTc interval $\cdot$ RR interval

\section{Introduction}

There are well known gender-related differences in human cardiac repolarization $[1,2]$. This is demonstrated by a longer heart-rate corrected QT (QTc) interval in women, which is the traditional clinical measurement for assessing the duration of ventricular repolarization [3]. Prolongation of ventricular repolarization may result in early after depolarizations, which 
in turn may induce re-entry and thereby provoke Torsade de Pointes and fatal ventricular arrhythmias [4-7].

The gender differences in the QTc interval are not present in young children, whereas at the time of onset of puberty the duration of the QTc interval in boys shortens, which results in a longer QTc interval in adult women compared to men [8-10]. These gender differences remain detectable until around the age of 50 years [10]. Since the period between puberty and 50 years of age coincides with the highest circulating levels of androgens in males, male sex hormones may play a role in the shorter QTc interval in men. Furthermore, virilized women exhibit QTc intervals similar to those of healthy men, whereas castrated men had QTc intervals similar to those of normal women [11]. Testosterone therapy also reduces QT dispersion in men with congestive heart failure [12].

These data suggest that testosterone might be an important regulator of ventricular repolarization, which might explain the gender-related differences in ventricular repolarization. Therefore, our aim was to study whether testosterone is associated with QTc, QT and RR interval duration. Since bio-available testosterone decreases with age, we studied this association in two cohorts. First, in a cohort of elderly (the Rotterdam study) and second, in a cohort with a younger mean age (the Study of Health In Pomerania (SHIP)) [13].

\section{Methods}

Setting and study design

\section{Rotterdam study}

The Rotterdam study is a prospective population-based cohort study, which started with a baseline visit between 1990 and 1993. The Medical Ethics Committee of the Erasmus Medical Center, Rotterdam, the Netherlands, approved the study. All inhabitants of Ommoord, a suburb of Rotterdam, aged 55 years and over, were invited to participate $(n=10,275)$. Of them, $7,983(78 \%)$ gave their written informed consent and took part in the baseline examination. Objectives and methods of the Rotterdam study have been described in detail elsewhere [14, 15]. At baseline, all participants were visited at home for a standardized questionnaire, and 7,151 were subsequently examined at the research center. The cohort is continuously monitored for major morbidity and mortality through linkage with general practitioner and municipality records. Drug prescriptions dispensed to participants by automated pharmacies are routinely stored in the database since January 1, 1991.

All male cohort members of the Rotterdam study ( $n=3,105)$, who had an ECG as well as serum testosterone and dehydroepiandrosterone sulfate (DHEAS) measurements at baseline were enrolled in the study population. Digitally stored ECGs were available for 2,200 male participants at the time of the first visit. Missing ECGs were mainly due to temporary technical problems with ECG recording. Androgen status was assessed in different random subsets of these males. Male participants with an ECG and with both a serum testosterone measurement and a DHEAS measurement $(n=540)$ were included. Participants who received anabolic steroids (anatomic therapeutical chemical (ATC) code A14A; $n=1$ ) were excluded. None of the participants received sexual hormones (ATC code G03), testosterone $5 \alpha$-reductase inhibitors (ATC code G04CB), sexual hormone antagonists (ATC code L02B) or had a pacemaker. Participants who used digoxin $(n=25)$, which is a QTc shortening agent, or QTc prolonging drugs $(n=6)$ as well as persons with evidence of left ventricular hypertrophy $(n=36)$ or left $(n=13)$ and right bundle branch block $(n=36)$ were excluded, since these conditions are associated with an altered QTc interval [16, 17]. Overall, 445 participants were included in the Rotterdam study population.

\section{The Study of Health In Pomerania}

The Study of Health In Pomerania is a cross-sectional population-based study in West Pomerania, a region in the northeast of Germany. The total population of West Pomerania selected for SHIP comprised 212,157 inhabitants. A two-stage cluster sampling method adopted from the WHO MONICA Project Augsburg, Germany yielded 12, 5 years age strata (20-79 years) for both genders, each including 292 individuals [18]. The sampling was performed from population registries where all German citizens are registered. Data collection started in October 1997 and was finished in March 2001. The net sample comprised 6,267 eligible subjects. Finally, 4,310 subjects (69\%) participated. The study was monitored by a board of independent scientists. All participants gave written informed consent. The study conformed to the principles of the Declaration of Helsinki as reflected by an a priori approval of the Ethics Committee of the University of Greifswald. Use of medication at baseline was recorded by a computeraided method using the ATC code.

All male cohort members of SHIP $(n=2,118)$ with ECG, testosterone and DHEAS measurements at baseline were enrolled in the study population. Digitally stored ECGs were available for 1,826 male participants at the time of the first visit. Male participants with an ECG and with both a testosterone and a DHEAS measurement $(n=1,677)$ were included. Participants who received sexual hormones (ATC code G03) $(n=3)$, testosterone $5 \alpha$ reductase inhibitors (ATC code $\mathrm{G} 04 \mathrm{CB} ; n=4$ ) or sexual 
hormone antagonists (ATC code L02B; $n=3$ ) were excluded. None of the participants reported to receive anabolic steroids (ATC code A14A). Furthermore, participants who used digoxin $(n=5)$ or digitoxin $(n=153)$, QTc prolonging drugs $(n=13)$, persons with a pacemaker ( $n=15$ ), as well as persons with evidence of left ventricular hypertrophy $(n=4)$ or left $(n=1)$ and right bundle branch block $(n=48)$ were excluded. Overall, 1,428 participants were included in the SHIP study population.

\section{QTc, QT and RR interval}

The endpoints of the study were the lengths of the QTc, QT and RR intervals in ms. A 12-lead resting ECG was recorded with an ACTA electrocardiograph (ESAOTE, Florence, Italy) at a sampling frequency of $500 \mathrm{~Hz}$ and stored digitally. All ECGs (both from the Rotterdam study as well as SHIP) were centrally processed in Rotterdam using the modular ECG analysis system (MEANS) to obtain ECG measurements, in agreement with the FDA guidance for clinical evaluation of QT/QTc interval prolongation [19]. The MEANS program has been evaluated and validated extensively [20-23]. In one of these validation studies, ECGs with selected abnormalities were analyzed by five cardiologists and 11 different computer programs of which MEANS performed as one of the best [23]. In a study in which QT intervals by manual measurement were compared with QT measurement by ECG machines, manual and automated measurements generated similar numerical results in three studies in healthy volunteers, which all included a positive control [24]. MEANS determines common onsets and offsets for all 12 leads together on one representative averaged beat, with the use of template matching techniques [21]. The QT interval is determined from the start of the QRS complex until the end of the $T$ wave. To adjust for heart rate, Bazett's formula $(\mathrm{QTc}=\mathrm{QT} / \sqrt{\mathrm{RR}})$ was used [25]. The RR interval was taken as the median of the RR intervals in the recording. Additionally, the MEANS program determines left ventricular hypertrophy and left and right bundle branch block.

\section{Steroids}

\section{Rotterdam study}

At baseline, non-fasting blood samples were obtained. Time of sampling was recorded. Testosterone and DHEAS were estimated using coated tube or double antibody RIAs, respectively, purchased from diagnostic systems laboratories [26].
SHIP

At baseline, non-fasting blood samples were obtained. Time of sampling was recorded. Testosterone and DHEAS were measured using competitive chemiluminescent enzyme immunoassays on an Immulite 2500 analyzer (DPC Biermann GmbH, Bad Nauheim, Germany).

Covariates

\section{Rotterdam study}

Hypertension was identified through the use of antihypertensive medication and/or the assessment of blood pressure measurements, according to the guidelines of the World Health Organisation [27]. Prevalence and incidence of myocardial infarction were assessed as previously described [28, 29]. Diabetes mellitus was defined as the use of blood glucose-lowering medication and/or a non-fasting serum glucose level of $11.1 \mathrm{mmol} / \mathrm{l}$ or higher and/or fasting serum glucose levels $\geq 7 \mathrm{mmol} / \mathrm{l}$ [30]. Prevalence and incidence of heart failure were assessed by the presence of suggestive signs and symptoms as previously described $[31,32]$. Potassium and calcium were measured by means of a Microlyte device. During the home interview, smoking status and use of alcohol were assessed. Creatinine clearance was computed with the Cockroft Gault method. Renal failure was defined by the internationally accepted criterion of a GFR below $60 \mathrm{ml} / \mathrm{min}$ [33]. Gamma-glutamyl transferase (GGT), aspartate-amino transferase (ASAT), alanine-amino transferase (ALAT) levels above the upper limit of normal were used to determine hepatic dysfunction.

\section{SHIP}

Hypertension was identified through the use of antihypertensive medication and/or the assessment of blood pressure measurements, according to the guidelines of the World Health Organisation [27]. Diabetes mellitus and myocardial infarction were defined as self-reported physician's diagnosis. Determination of calcium was performed by a colorimetric assay and potassium by ionselective electrodes (Roche/Hitachi 717; Roche Diagnostics GmbH, Mannheim, Germany). During the home interview, smoking status and use of alcohol were assessed. Creatinine clearance was computed with the Cockroft Gault method. Renal failure was defined by the internationally accepted criterion of a GFR below $60 \mathrm{ml} / \mathrm{min}$ [33]. GGT, ALAT and ASAT were used to determine hepatic dysfunction. 
Statistical analysis

The association between the QTc, QT, RR intervals and testosterone was assessed through linear regression with log-transformed testosterone and testosterone measurements divided in tertiles. Since DHEAS was associated with both the QTc and the RR interval, we adjusted all analyses for DHEAS. Furthermore, all analyses were adjusted for age, time of blood withdrawal (recorded in hours and minutes), hypertension, myocardial infarction, diabetes mellitus, potassium, calcium and in the Rotterdam study also for heart failure.

First, a linear regression analysis was conducted with QTc, QT (adjusted for the length of the RR interval) and $\mathrm{RR}$ interval as dependent and testosterone as independent variables for the Rotterdam study and SHIP separately. Second, we conducted a pooled analysis of the Rotterdam study and SHIP. Furthermore, we performed several sensitivity analyses: first, by stratification for age. Second, in additional analyses we adjusted for smoking, alcohol abuse, renal failure, hepatic failure and use of beta-blockers. All analyses were performed using SPSS for Windows version 15.0 (Chicago, Illinois, USA).

\section{Results}

\section{Subject characteristics}

The baseline characteristics of the participants are presented in Table 1. The mean age of the study population of the Rotterdam study was 68.0 years [standard deviation (SD) 7.8 years]. The mean testosterone level was $11.3 \mathrm{nmol} / \mathrm{l}(\mathrm{SD}=3.7)$. The mean age of the study population of SHIP was 49.1 years (SD 16.0 years). Mean testosterone levels were $17.0 \mathrm{nmol} / \mathrm{l}(\mathrm{SD}=5.9)$.

Androgens and QTc interval

The mean duration of the QTc interval was approximately similar in both studies, the mean duration of the QT and RR interval was slightly longer in the Rotterdam study than in SHIP (Table 1). Comparison of the highest testosterone tertile with the lowest tertile showed no significant association between testosterone and QTc [-5.9 ms $(95 \%$ CI- $13.8 ; 2.1)$ and $-2.9 \mathrm{~ms}(95 \%$ CI- 5.9; 0.1) respectively] nor with the QT interval [-2.4 ms $(95 \%$ CI- 8.8; 4.0) and $-0.6 \mathrm{~ms}(95 \% \mathrm{CI}-3.0 ; 1.8)$ respectively] in the Rotterdam study and SHIP separately (Table 2). The logarithmic transformation of testosterone was associated with the QTc interval in SHIP [-7.9 ms (95\% CI- 15.3; -0.6)].

In SHIP, the RR interval increased gradually with increasing testosterone levels ( $P$ value for trend 0.004$)$ and
Table 1 Baseline characteristics of study population

\begin{tabular}{lll}
\hline & Rotterdam study & SHIP \\
\hline Number of participants & 445 & 1,428 \\
Age (years, mean, SD) & $68.0(7.8)$ & $49.1(16.0)$ \\
Mean QTc interval (ms; SD) & $423.2(25.0)$ & $423.5(24.4)$ \\
Mean QT interval (ms; SD) & $398.2(28.6)$ & $394.1(29.9)$ \\
Mean RR interval (ms; SD) & $896.2(156.7)$ & $876.0(156.2)$ \\
Body mass index (kg/m²; SD) & $26.6(3.4)$ & $27.7(4.2)$ \\
Diabetes mellitus $(\mathrm{n}, \%)$ & $50(11.2)$ & $95(6.7)$ \\
Myocardial infarction $(\mathrm{n}, \%)$ & $84(18.9)$ & $59(4.1)$ \\
Hypertension (n, \%) & $111(24.9)$ & $594(41.6)$ \\
Heart failure (n, \%) & $7(1.6)$ & - \\
Mean potassium $(\mathrm{mmol} / \mathrm{l} ; \mathrm{SD})$ & $4.1(0.3)$ & $4.1(0.3)$ \\
Mean calcium $(\mathrm{mmol} / \mathrm{l} ; \mathrm{SD})$ & $2.4(0.1)$ & $2.4(0.1)$ \\
Mean DHEAS $(\mu \mathrm{mol} / \mathrm{l} ; \mathrm{SD})$ & $4.3(2.7)$ & $5.3(3.3)$ \\
Mean testosterone $(\mathrm{nmol} / \mathrm{l} ; \mathrm{SD})$ & $11.3(3.7)$ & $17.0(5.9)$ \\
\hline
\end{tabular}

$S D$ standard deviation

DHEAS dehydroepiandrosterone sulfate

the second and third tertiles of testosterone had longer RR intervals compared to the first tertile [24.0 ms (95\% CI 3.8; $44.1)$ and $31.3 \mathrm{~ms}(95 \%$ CI $10.3 ; 52.3)$ respectively]. The logarithmic transformation of testosterone was associated with the RR interval in both the Rotterdam study and SHIP [149.8 ms (95\% CI 30.6; 269.1) and $55.9 \mathrm{~ms}$ (95\% CI 5.2; 106.6) respectively].

\section{Pooled analysis}

There was a gradual decrease of the QTc interval among the tertiles ( $P$ value for trend 0.024$)$, while the third tertile of testosterone was significantly associated with the QTc interval compared to the first tertile $[-3.4 \mathrm{~ms}(95 \%$ CI- 6.5 ; -0.3); Table 3]. Compared to the first tertile of testosterone, the third tertile was not associated with the QT interval $[-0.7 \mathrm{~ms}$ (95\% CI- 3.1; 1.8)].

There was a gradual increase of the RR interval among the tertiles ( $P$ value for trend 0.002 ), while the third tertile of testosterone was significantly associated with the RR interval compared to the first tertile [33.5 ms (95\% CI 12.2; 54.8)]. Sensitivity analyses revealed no effect modification by age. Additional adjustment for smoking, alcohol, renal failure, hepatic failure or use of beta-blockers did not change the estimates.

\section{Discussion}

In this pooled analysis of two population-based studies, we found that serum testosterone levels were associated with shortening of the QTc interval and prolongation of the RR 
Table 2 Association of testosterone with QTc, QT and RR interval

\begin{tabular}{|c|c|c|c|c|}
\hline & Testosterone & $\begin{array}{l}\text { QTc-interval in ms } \\
(95 \% \mathrm{CI})^{\mathrm{a}}\end{array}$ & $\begin{array}{l}\text { QT-interval in ms } \\
(95 \% \mathrm{CI})^{\mathrm{b}}\end{array}$ & $\begin{array}{l}\text { RR-interval in ms } \\
(95 \% \mathrm{CI})^{\mathrm{a}}\end{array}$ \\
\hline \multirow[t]{6}{*}{ Rotterdam study $(n=445)$} & Log-transformation & $-6.5(-23.7 ; 10.8)$ & $5.9(-8.2 ; 20.1)$ & $149.8(30.6 ; 269.1)$ \\
\hline & Tertiles & & & \\
\hline & 1 & Reference & Reference & Reference \\
\hline & 2 & $3.8(-4.1 ; 11.7)$ & $5.5(-0.8 ; 11.9)$ & $29.2(-27.3 ; 85.8)$ \\
\hline & 3 & $-5.9(-13.8 ; 2.1)$ & $-2.4(-8.8 ; 4.0)$ & $42.4(-14.3 ; 99.2)$ \\
\hline & $P$ value for linear trend & 0.132 & 0.419 & 0.143 \\
\hline \multirow[t]{6}{*}{$\operatorname{SHIP}(n=1,428)$} & Log-transformation & $-7.9(-15.3 ;-0.6)$ & $-3.4(-9.2 ; 2.4)$ & $55.9(5.2 ; 106.6)$ \\
\hline & Tertiles & & & \\
\hline & 1 & Reference & Reference & Reference \\
\hline & 2 & $-0.7(-3.6 ; 2.2)$ & $1.5(-0.8 ; 3.9)$ & $24.0(3.8 ; 44.1)$ \\
\hline & 3 & $-2.9(-5.9 ; 0.1)$ & $-0.6(-3.0 ; 1.8)$ & $31.3(10.3 ; 52.3)$ \\
\hline & $P$ value for linear trend & 0.061 & 0.623 & 0.004 \\
\hline
\end{tabular}

CI confidence interval

${ }^{a}$ Adjusted for age, time of blood withdrawal, diabetes, hypertension, myocardial infarction, potassium, calcium, BMI, dehydroepiandrosterone sulfate (DHEAS) and in the Rotterdam Study also for heart failure

b Adjusted for age, time of blood withdrawal, diabetes, hypertension, myocardial infarction, potassium, calcium, BMI, DHEAS, RR interval and in the Rotterdam study also for heart failure

Testosterone in nmol/1. For the Rotterdam study the tertiles were defined as (1) $\leq 9.9$; (2) $10.0-12.6$; (3) $\geq 12.7$. For SHIP the tertiles were defined as $(1) \leq 13.9$; (2) $14.0-18.6$; (3) $\geq 18.7$

The bold values indicate significance at $P<0.05$

Table 3 Association of testosterone with QTc, QT and interval: pooled analysis of the Rotterdam study and SHIP $(n=1,873)$

\begin{tabular}{|c|c|c|c|}
\hline Testosterone & QTc-interval in ms $(95 \% \mathrm{CI})^{\mathrm{a}}$ & QT-interval in ms $(95 \% \mathrm{CI})^{\mathrm{b}}$ & RR-interval in ms $(95 \% \mathrm{CI})^{\mathrm{a}}$ \\
\hline Log-transformation & $-8.1(-14.8 ;-1.4)$ & $-3.1(-8.4 ; 2.3)$ & $62.5(15.7 ; 109.2)$ \\
\hline \multicolumn{4}{|l|}{ Tertiles } \\
\hline 1 & Reference & Reference & Reference \\
\hline 2 & $-0.7(-3.6 ; 2.1)$ & $0.5(-1.8 ; 2.8)$ & $10.8(-9.1 ; 30.8)$ \\
\hline 3 & $-3.4(-6.5 ;-0.3)$ & $-0.7(-3.1 ; 1.8)$ & $33.5(12.2 ; 54.8)$ \\
\hline$P$ value for linear trend & 0.024 & 0.529 & 0.002 \\
\hline
\end{tabular}

CI confidence interval

${ }^{a}$ Adjusted for age, time of blood withdrawal, diabetes, hypertension, myocardial infarction, potassium, calcium, BMI, dehydroepiandrosterone sulfate (DHEAS) and cohort

b Adjusted for age, time of blood withdrawal, diabetes, hypertension, myocardial infarction, potassium, calcium, BMI, RR interval, DHEAS and cohort

Testosterone in nmol/l. The tertiles were defined as (1) $\leq 12.5$; (2) $12.6-17.3$; (3) $\geq 17.4$

The bold values indicate significance at $P<0.05$

interval in men, whereas we did not find an association between serum testosterone levels and the uncorrected QT interval, which could be due to a lack of power. Earlier, it has been suggested that the shorter QTc interval in men than in women may be explained by the fact that testosterone influences repolarization by its effect on $\mathrm{Ca}^{2+}$ and $\mathrm{K}^{+}$channels, as well as on hERG $\mathrm{K}^{+}$channels [34]. Since the QT interval was not significantly associated with serum testosterone, our findings could suggest that the difference in the QTc interval in men is mainly due to the underlying association with the RR interval. Since QTc is calculated by Bazett's formula, an increase in RR interval will lead to a shorter QTc interval. However, the QTc interval calculated by Bazett's formula is clinically relevant and is often used in epidemiological and clinical investigations. The QTc interval has been associated with an increased risk of all-cause mortality and sudden cardiac death $[1,2]$. In addition, low testosterone levels are associated with an increased risk of all-cause mortality in men, [3] which is consistent with our findings of an increased QTc interval 
and decreased RR interval in males with low testosterone levels. The use of testosterone supplementation is increasing, [4] in spite of limited knowledge on benefits and long-term safety and therefore it is important to know the effects of endogenous testosterone levels.

The gender differences in the QTc interval remain detectable until around the age of 50 years [10], this could be due to the fact that bio-available testosterone decreases with age [13]. In SHIP and the pooled analyses the direction of change was as expected for the QTc and RR-adjusted QT interval. In the Rotterdam study, however, the direction of change differed, which could be due to limited power. In addition, we did not find a significant association with the RR interval in the Rotterdam study, which could also be due to limited power. It is well known that women have a longer QTc interval than men, [2] whereas the uncorrected QT intervals are rather similar in men and women [10]. Since women have a faster heart rate, and therefore shorter RR intervals compared to men, women have a prolonged QTc interval when the same heart rate correction formula is applied. Recently it has been demonstrated in eleven hypogonadic men, that the difference in the heart-rate-independent QT interval duration is dependent on testosterone levels [35]. The gender differences in the RR interval remain after autonomic blockade, which suggests a possible gender-related difference in sino-atrial node function [36]. However, this difference appears to be related to a difference in maximum exercise capacity between men and women rather than to intrinsic sinus node gender-related differences [36]. Since androgen levels increase in response to exercise [37], the increased RR interval in men with high testosterone levels could be due to increased exercise capacity, which affects the sino-atrial node function.

Our investigation has several strengths that include the population-based design of participating studies and the central ECG data management, which facilitates best comparability between both studies. Although we did not use the complete population in the Rotterdam study, selection bias is unlikely because the characteristics of the study population were comparable to the baseline characteristics of the whole population of the Rotterdam study. Furthermore, information bias is unlikely because data were gathered prospectively and because the use of digital ECG recordings all measured using the automatic MEANS system likely reduced intra- and interobserver variability in the assessment of the QTc, QT and RR intervals. Confounding was minimized by adjusting for most known risk factors. However, our study has also some limitations. Because of the cross-sectional design we cannot exclude that QTc shortening or RR interval prolongation was already present in some participants before the decrease of testosterone. Several measurements within a person would be needed to assess intra-individual differences. Therefore, the results from this study should be confirmed with longitudinal data from other large cohorts. Another limitation of our study is that repeated blood sampling in men with initially low serum testosterone levels is recommended to confirm androgen deficiency [38], but this and other epidemiologic studies [39-42] were based on a single measurement of serum testosterone. In addition, two different methods were used to measure testosterone levels. However, as can be expected because of the higher average age, testosterone levels were somewhat lower in the Rotterdam study (Table 1), which is in agreement with the fact that testosterone levels decrease with age [5]. The results of the pooled analyses did not substantially change after adjustment for age. In addition, the direction of change of the QTc and RR interval were comparable and as expected (Table 2).

However, misclassification of men with low serum testosterone levels would be expected to underestimate, not overestimate associations.

\section{Conclusion}

We demonstrated in two population-based studies that serum testosterone is associated with shortening of the QTc interval and prolongation of the RR interval in men, whereas we did find a minor non-significant association between serum testosterone levels and the QT interval, possibly due to a lack of power. The fact that the strongest decrease was in the clinically relevant QTc interval in men is partly explained by the effect of RR-prolongation in the denominator of Bazett's formula for the QTc-interval.

Acknowledgments SHIP is part of the Community Medicine Research net (CMR) of the University of Greifswald, Germany, which is funded by the Federal Ministry of Education and Research (grant no. ZZ9603), the Ministry of Cultural Affairs as well as the Social Ministry of the Federal State of Mecklenburg-West Pomerania (http://ship.community-medicine.de/).

Open Access This article is distributed under the terms of the Creative Commons Attribution Noncommercial License which permits any noncommercial use, distribution, and reproduction in any medium, provided the original author(s) and source are credited.

\section{References}

1. Merri M, Benhorin J, Alberti M, Locati E, Moss AJ. Electrocardiographic quantitation of ventricular repolarization. Circulation. 1989;80(5):1301-8.

2. Bazett $H$. An analysis of the time realtionship of electrograms. Heart. 1920;7:353-70.

3. Abi-Gerges N, Philp K, Pollard C, Wakefield I, Hammond TG, Valentin JP. Sex differences in ventricular repolarization: from 
cardiac electrophysiology to Torsades de Pointes. Fundam Clin Pharmacol. 2004;18(2):139-51.

4. Lasser KE, Allen PD, Woolhandler SJ, Himmelstein DU, Wolfe SM, Bor DH. Timing of new black box warnings and withdrawals for prescription medications. Jama. 2002;287(17):2215-20.

5. Roden DM. Drug-induced prolongation of the QT interval. N Engl J Med. 2004;350(10):1013-22.

6. Al-Khatib SM, LaPointe NM, Kramer JM, Califf RM. What clinicians should know about the QT interval. Jama. 2003; 289(16):2120-7.

7. Yap YG, Camm AJ. Drug induced QT prolongation and torsades de pointes. Heart. 2003;89(11):1363-72.

8. McCammon R. A longitudinal study of electrocardiographic intervals in healthy children. Acta Pediatr. 1960;50(126):3-54.

9. Stramba-Badiale M, Spagnolo D, Bosi G, Schwartz PJ. Are gender differences in QTc present at birth? MISNES investigators. Multicenter Italian study on neonatal electrocardiography and sudden infant death syndrome. Am J Cardiol. 1995;75(17): 1277-8.

10. Rautaharju PM, Zhou SH, Wong S, Calhoun HP, Berenson GS, Prineas R, et al. Sex differences in the evolution of the electrocardiographic QT interval with age. Can J Cardiol. 1992;8(7): 690-5.

11. Bidoggia H, Maciel JP, Capalozza N, Mosca S, Blaksley EJ, Valverde E, et al. Sex differences on the electrocardiographic pattern of cardiac repolarization: possible role of testosterone. Am Heart J. 2000;140(4):678-83.

12. Malkin CJ, Morris PD, Pugh PJ, English KM, Channer KS. Effect of testosterone therapy on QT dispersion in men with heart failure. Am J Cardiol. 2003;92(10):1241-3.

13. Ferrini RL, Barrett-Connor E. Sex hormones and age: a crosssectional study of testosterone and estradiol and their bioavailable fractions in community-dwelling men. Am J Epidemiol. 1998;147(8):750-4.

14. Hofman A, Grobbee DE, de Jong PT, van den Ouweland FA. Determinants of disease and disability in the elderly: the Rotterdam elderly study. Eur J Epidemiol. 1991;7(4):403-22.

15. Hofman A, Breteler MM, van Duijn CM, Krestin GP, Pols HA, Stricker BH, et al. The Rotterdam study: objectives and design update. Eur J Epidemiol. 2007;22(11):819-29.

16. Kulan K, Ural D, Komsuoglu B, Agacdiken A, Goldeli O, Komsuoglu SS. Significance of QTc prolongation on ventricular arrhythmias in patients with left ventricular hypertrophy secondary to essential hypertension. Int J Cardiol. 1998;64(2): 179-84.

17. Piotrowicz K, Zareba W, McNitt S, Moss AJ. Repolarization duration in patients with conduction disturbances after myocardial infarction. Am J Cardiol. 2007;99(2):163-8.

18. Keil U, Liese AD, Hense HW, Filipiak B, Doring A, Stieber J, et al. Classical risk factors and their impact on incident non-fatal and fatal myocardial infarction and all-cause mortality in southern Germany. Results from the MONICA Augsburg cohort study 1984-1992. Monitoring Trends and Determinants in Cardiovascular Diseases. Eur Heart J. 1998;19(8):1197-207.

19. ICH Topic E14: The Clinical Evaluation of QT/QTe interval prolongation and proarrhythmic potential for non-antiarrhythmic drugs. (CHMP/ICH/2/04) November 2005.

20. de Bruyne MC, Kors JA, Hoes AW, Kruijssen DA, Deckers JW, Grosfeld M, et al. Diagnostic interpretation of electrocardiograms in population-based research: computer program research physicians, or cardiologists? J Clin Epidemiol. 1997;50(8):947-52.

21. van Bemmel JH, Kors JA, van Herpen G. Methodology of the modular ECG analysis system MEANS. Methods Inf Med. 1990;29(4):346-53.

22. Willems JL, Abreu-Lima C, Arnaud P, van Bemmel JH, Brohet $\mathrm{C}$, Degani R, et al. The diagnostic performance of computer programs for the interpretation of electrocardiograms. N Engl J Med. 1991;325(25):1767-73.

23. Willems JL, Arnaud P, van Bemmel JH, Bourdillon PJ, Degani R, Denis B, et al. A reference data base for multilead electrocardiographic computer measurement programs. J Am Coll Cardiol. 1987;10(6):1313-21.

24. Darpo B, Agin M, Kazierad DJ, Layton G, Muirhead G, Gray P, et al. Man versus machine: is there an optimal method for QT measurements in thorough QT studies? J Clin Pharmacol. 2006; 46(6):598-612.

25. Bazett HC. The time relations of the blood-pressure changes after excision of the adrenal glands, with some observations on blood volume changes. J Physiol. 1920;53(5):320-39.

26. Diagnostic Systems Laboratories (DSL): Webster, Texas, available at http://www.dslabs.com. 2007.

27. WHO. World Health Organization-international society of hypertension guidelines for the management of hypertension. Guidelines subcommittee. J Hypertens. 1999;17(2):151-83.

28. Bots ML, Hoes AW, Koudstaal PJ, Hofman A, Grobbee DE. Common carotid intima-media thickness and risk of stroke and myocardial Infarction : The Rotterdam study. Circulation. 1997; 96(5):1432-7.

29. Vliegenthart R, Oudkerk M, Song B, van der Kuip DA, Hofman A, Witteman JC. Coronary calcification detected by electronbeam computed tomography and myocardial infarction. The Rotterdam coronary calcification study. Eur Heart J. 2002;23(20): 1596-603.

30. Diabetes Mellitus: Technical Reports series 894. Geneva: World Health Organisation; 1985.

31. Bleumink GS, Knetsch AM, Sturkenboom MC, Straus SM, Hofman A, Deckers JW, et al. Quantifying the heart failure epidemic: prevalence, incidence rate, lifetime risk and prognosis of heart failure The Rotterdam study. Eur Heart J. 2004; 25(18):1614-9.

32. Mosterd A, Hoes AW, de Bruyne MC, Deckers JW, Linker DT, Hofman A, et al. Prevalence of heart failure and left ventricular dysfunction in the general population; The Rotterdam study. Eur Heart J. 1999;20(6):447-55.

33. Levey AS, Eckardt KU, Tsukamoto Y, Levin A, Coresh J, Rossert J, et al. Definition and classification of chronic kidney disease: a position statement from kidney disease: improving global outcomes (KDIGO). Kidney Int. 2005;67(6):2089-100.

34. Ridley JM, Shuba YM, James AF, Hancox JC. Modulation by testosterone of an endogenous hERG potassium channel current. J Physiol Pharmacol. 2008;59(3):395-407.

35. Charbit B, Christin-Maitre S, Demolis JL, Soustre E, Young J, Funck-Brentano C. Effects of testosterone on ventricular repolarization in hypogonadic men. Am J Cardiol. 2009;103(6): 887-90.

36. Burke JH, Goldberger JJ, Ehlert FA, Kruse JT, Parker MA, Kadish AH. Gender differences in heart rate before and after autonomic blockade: evidence against an intrinsic gender effect. Am J Med. 1996;100(5):537-43.

37. Tremblay MS, Copeland JL, Van Helder W. Effect of training status and exercise mode on endogenous steroid hormones in men. J Appl Physiol. 2004;96(2):531-9.

38. Bhasin S, Cunningham GR, Hayes FJ, Matsumoto AM, Snyder PJ, Swerdloff RS, et al. Testosterone therapy in adult men with androgen deficiency syndromes: an endocrine society clinical practice guideline. J Clin Endocrinol Metab. 2006;91(6):19952010.

39. Araujo AB, Kupelian V, Page ST, Handelsman DJ, Bremner WJ, McKinlay JB. Sex steroids and all-cause and cause-specific mortality in men. Arch Intern Med. 2007;167(12):1252-60.

40. Khaw KT, Dowsett M, Folkerd E, Bingham S, Wareham N, Luben R, et al. Endogenous testosterone and mortality due to all 
causes, cardiovascular disease, and cancer in men: European prospective investigation into cancer in Norfolk (EPIC-Norfolk) Prospective Population Study. Circulation. 2007;116(23): 2694-701.

41. Laughlin GA, Barrett-Connor E, Bergstrom, J.: Low serum testosterone and mortality in older men. The Journal of clinical endocrinology and metabolism. 2007 Oct 2.
42. Smith GD, Ben-Shlomo Y, Beswick A, Yarnell J, Lightman S, Elwood P. Cortisol, testosterone, and coronary heart disease: prospective evidence from the Caerphilly study. Circulation. 2005;112(3):332-40. 